\title{
A BOOTSTRAP APPROACH TO TESTING FOR SYMMETRY IN THE GRANGER AND LEE ASYMMETRIC ERROR CORRECTION MODEL
}

\author{
Henry De-Graft Acquah, Senior Lecturer \\ Department of Agricultural Economics and Extension \\ University of Cape Coast, Cape Coast, Ghana \\ Phone: +00233245543956, E-mail: henrydegraftacquah@yahoo.com
}

Received October 11, 2012

\begin{abstract}
The power of the Granger and Lee (1989) model of asymmetry is examined via bootstrap simulation. The results of the bootstrap simulation indicate that the Granger and Lee model has low power in rejecting the null hypothesis of symmetric adjustments. The power of the test depends on the bootstrap sample size, difference in adjustment speeds and the amount of noise in the data generating process used in the application. With a small bootstrap sample and large noise level the Granger and Lee model display low power in rejecting the null hypothesis of symmetry.
\end{abstract}

\section{KEY WORDS}

Bootstrap Methods; Asymmetric Adjustments; Power test; Granger and Lee Model.

Granger and Lee (1989) propose a methodology to investigate asymmetric adjustment in economic time series. This entails specifying a cointegrating relationship between a set of variables and allowing the speed of adjustment of the endogenous variable to depend on whether its current deviation from the equilibrium value indicated by the cointegrating vector is positive or negative. In recent studies, the Granger and Lee error correction approach has been applied to a variety of different problems and found to have low power in rejecting the null hypothesis of symmetric adjustment. Acquah et al (2009) and Cook et al $(1999 ; 2000 ; 2003)$ sheds light on power of the Granger and Lee model in rejecting the null hypothesis of symmetric adjustments using Monte Carlo methods but did not consider the use of bootstrap methods to analyse the power of the Granger and Lee model in rejecting the null hypothesis of symmetric adjustments.

Application of bootstrap methods to construct new samples which are based on original data gives an advantage over the previous Monte Carlo methods which makes implicit assumption about the true values of the parameters. Regardless of the robustness of boostrap methods, little work has been done to investigate power of the Granger and Lee model in rejecting the null hypothesis of symmetric adjustments in bootstrap samples.
A fundamental question which remains unanswered is how well Granger and Lee model will perform in rejecting the null hypothesis of symmetric adjustments in bootstrap samples. As sample size increases, will the Granger and Lee model have increased power as noted in previous Monte Carlo studies?

This study therefore aims empirically to investigate the power of the Granger and Lee model in rejecting the null hypothesis of symmetric adjustments in bootstrap samples. This contributes to understanding the power of Granger and Lee model and their empirical performance in asymmetric price transmission power analysis. Furthermore, this study explores the bootstrap methods as an alternative framework to the Monte Carlo Methods and demonstrates their usefulness in power analysis of the Granger and Lee asymmetric error correction model.

The rest of the paper proceeds as follows: In the following section, an introduction of the Granger and Lee test for asymmetry is presented. This is followed by an introduction of bootstrap methods. A practical application in which the performance of the Granger and Lee model in rejecting the null hypothesis of symmetric adjustments is evaluated and the results of the Bootstrap simulations are presented. Finally, the study ends with conclusions. 
Granger and Lee Asymmetric Model. The Granger and Lee model have been extensively used in evaluating asymmetric price transmission in agricultural markets. Von CramonTaubadel (1998) using the Granger and Lee model, demonstrates that transmission between producer and wholesale pork prices in northern Germany is asymmetric. Acquah (2010) applica-

$$
\Delta y_{t}=\beta_{1} \Delta x_{t}+\beta_{2}(y-x)_{t-1}+\varepsilon_{2, t} \quad \varepsilon_{2, t} \sim N\left(0, \delta^{2}\right)
$$

where $\mathrm{y}$ and $\mathrm{x}$ are price series. If $\mathrm{y}$ and $\mathrm{x}$ are typically $\mathrm{I}(1)$ processes that are cointegrated, then there exists an equilibrium relationship between $y$ and $\mathrm{x}$ which is defined by an error correction term. The long run dynamics captured by the er-

$$
\begin{aligned}
& (y-x)_{t}^{+}=\left[\begin{array}{cc}
(y-x)_{t}, & \text { if }(y-x)_{t}>0 \\
\text { zero } & \text { otherwise }
\end{array}\right. \\
& (y-x)_{t}^{-}=\left[\begin{array}{cc}
(y-x)_{t}, & \text { if }(y-x)_{t}<0 \\
\text { zero } & \text { otherwise }
\end{array}\right.
\end{aligned}
$$

The resulting asymmetric model is specified as

$$
\Delta y_{t}=\beta_{1} \Delta x_{t}+\beta_{2}^{+}(y-x)_{t-1}^{+}+\beta_{2}^{-}(y-x)_{t-1}^{-}+\varepsilon_{3, t} \quad \varepsilon_{3, t} \sim N\left(0, \delta^{2}\right)
$$

Equation 4 is referred to as the Granger and Lee asymmetric model. Asymmetry is incorporated by allowing the speed of adjustment to differ for the positive and negative components of the Error Correction Term (ECT) since the long run relationship captured by the ECT was implicitly symmetric. Symmetry in equation (4) is tested by determining whether the coefficients $\left(\beta_{2}{ }^{+}\right.$and $\left.\beta_{2}{ }^{-}\right)$ are identical (that is $H_{0}: \beta_{2}{ }^{+}=\beta_{2}^{-}$).

The Bootstrap. Bootstrapping involves repeated random sampling with replacement from the original data, to produce random samples of the same size of the original sample. Each resampled data is referred to as a bootstrap sample. Each bootstrap sample can be used to estimate a parameter of interest. The «with replacement» means that any observation can be sampled more than once in each bootstrap sample. This is essential since sampling without replacement would simply lead to a random permutation of the original data with the statistic of interest being the same. Replicating the process, a larger number of times provides the required information on the variability of the estimator of interest.
Parametric Bootstrap. Parametric bootstrapping refers to the process of resampling from the residuals of a parametric model. In this case, the regression model is first estimated and a bootstrap sample of the residuals is then drawn. These residuals are then added to the original regression equation (and $\mathrm{x}$ values) to generate new bootstrap values for the outcome variable. Ordinary least squares are then used to estimate the new bootstrap regression coefficients, for this bootstrap sample. This process of resampling of the residuals, adding them to the fitted values and estimating the regression coefficients is repeated lots of times to estimate the parameters of interest from the bootstrap samples. In summary, the bootstrap procedure can be outline in 3 steps as follows:

1. Generate $\varepsilon^{*}$ by sampling with replacement from $\hat{\varepsilon}_{1}, \ldots ., \hat{\varepsilon}_{n}$

2. Form $y^{*}=X \hat{\beta}+\varepsilon^{*}$

3. Compute $X \hat{\beta}^{*}$ from $\left(X, y^{*}\right)$

Analysis of the Power of the Test for Asymmetry: Bootstrap Simulation Results. In order to investigate the power of the test for asymmetry under various conditions, a series of Bootstrap comparison of the Granger and Lee model 
is carried out based on 10000 replications. In the spirit of Holly et al. (2003), the data generation process is specified in equation (4) with $\beta_{1}$ set to 0.5 and $\left(\beta_{2}{ }^{+}, \beta_{2}^{-}\right) \in(-0.25,-0.50)$ or $(-0.25,-0.75)$. Specifically, the power of the Granger and Lee model is evaluated under conditions of different sample sizes, noise levels and two levels of asymmetry given by $\left(\beta_{2}{ }^{+}, \beta_{2}{ }^{-}\right) \in(-0.25,-0.50)$ or $(-0.25,-0.75)$.

Fundamentally, subtle and strong levels of asymmetry are considered in the data generating process. The Granger and Lee model is evaluated in terms of its ability to reject the (false) null hypothesis of symmetric adjustment against the (true) alternative of asymmetric adjustment using an F-test of the restricted versus the unrestricted model.

The Bootstrap simulation results indicate the low power of the conventional F-test in rejecting the null of symmetric adjustment in small bootstrap sample sizes. There is some improvement in power when the amount of noise in the data generating process (DGP) is decreased systematically. Similarly, when the difference in asymmetric adjustment parameters is increased from 0.25 to 0.50 in the true model, an increase in power is also observed in Granger and Lee model as illustrated in Table 1. However, it is only when the bootstrap sample size is increased to 500 that a reasonable result is obtained.

Table 1 - Rejection frequencies based on 10000 Bootstrap replications

\begin{tabular}{|c|c|c|c|c|}
\hline \multicolumn{5}{|c|}{ Granger and Lee-Error Correction Model DGP(GL-ECM) } \\
\hline Sample size & $\left(\beta_{2}^{+}, \beta_{2}^{-}\right)$ & Error Size & Rejection & Frequencies \\
\hline 50 & $(-0.25,-0.50)$ & 3 & 0.1193 & 0.0382 \\
\hline 50 & $(-0.25,-0.50)$ & 2 & 0.1215 & 0.0438 \\
\hline 50 & $(-0.25,-0.50)$ & 1 & 0.1693 & 0.0684 \\
\hline 150 & $(-0.25,-0.50)$ & 3 & 0.1547 & 0.0546 \\
\hline 150 & $(-0.25,-0.50)$ & 2 & 0.1795 & 0.0728 \\
\hline 150 & $(-0.25,-0.50)$ & 1 & 0.3538 & 0.1924 \\
\hline 500 & $(-0.25,-0.50)$ & 3 & 0.2206 & 0.0982 \\
\hline 500 & $(-0.25,-0.50)$ & 2 & 0.3289 & 0.1729 \\
\hline 500 & $(-0.25,-0.50)$ & 1 & 0.7438 & 0.5516 \\
\hline 50 & $(-0.25,-0.75)$ & 3 & 0.1409 & 0.0447 \\
\hline 50 & $(-0.25,-0.75)$ & 2 & 0.1704 & 0.0744 \\
\hline 50 & $(-0.25,-0.75)$ & 1 & 0.3594 & 0.1847 \\
\hline 150 & $(-0.25,-0.75)$ & 3 & 0.2229 & 0.1143 \\
\hline 150 & $(-0.25,-0.75)$ & 2 & 0.3494 & 0.1905 \\
\hline 150 & $(-0.25,-0.75)$ & 1 & 0.7698 & 0.5973 \\
\hline 500 & $(-0.25,-0.75)$ & 3 & 0.4606 & 0.2849 \\
\hline 500 & $(-0.25,-0.75)$ & 2 & 0.7366 & 0.5486 \\
\hline 500 & $(-0.25,-0.75)$ & 1 & 0.9969 & 0.9872 \\
\hline
\end{tabular}

In summary, the bootstrap sample sizes, difference between the asymmetric adjustment parameters and the amount of noise in the data generating process are important in the power of the test for asymmetry. With small bootstrap sample size or large noise, the Granger and Lee model display low power in rejecting the (false) null hypothesis of symmetry. Fundamentally, I have shown that bootstrap techniques also offer a framework for evaluating the power of the test for asymmetry.

Numerous studies have evaluated the power of the Granger and Lee model in rejecting the null hypothesis of symmetric adjustment using Monte Carlo methods. In the present study, the power of the Granger and Lee Model has been evaluated using bootstrap methods. An important feature of the bootstrap simulation results is that they generally echo the results from existing Monte Carlo studies. Acquah (2009)'s Monte Carlo experimentation shows that the power of the Granger and Lee model has low power in rejecting the null hypothesis of symmetric adjustments. The Monte Carlo studies further noted that it was only in large samples that a reasonable result was obtained. Similarly, the Monte Carlo experimentation results of Cook $(1999 ; 2000)$ also demonstrated the low power of the Granger and Lee model in small samples. The present bootstrap simulation results and the previous Monte Carlo Studies all point to the fact that the power of the Granger and Lee model depends on sample size, difference between the asymmetric 
adjustment parameters and the amount of noise in the data.

Conclusions. The power of the Granger and Lee approach in rejecting the null hypothesis of symmetric adjustments in bootstrap samples has been evaluated. In particular, it has been demonstrated that the power of the Granger and Lee model depends on various conditions or design characteristics. The results of the Bootstrap simulations indicate that rejection frequencies increase with increases in bootstrap sample size, increases in the difference between the asymmetric adjustment speeds and decreases in the amount of noise in the true data generating process used in the application. Furthermore, this study has demonstrated the usefulness of the bootstrap methods as an alternative framework for evaluating the power of the test of asymmetry. The results contribute to knowledge and understanding of the various conditions which improves the power of the Granger and Lee test for asymmetry in bootstrap samples. Researchers modeling asymmetry can derive from this research how to implement the Bootstrap methodology in testing the power of their models in an asymmetric price transmission context.

\section{REFERENCES}

Acquah, H.D. (2010). Asymmetry in RetailWholesale Price Transmission for Maize: Evidence from Ghana in American Euroasian Journal of Agricultural and Environmental Science, , 7(4):452-456, 2010

Acquah, H.D. and Von Cramon-Taubadel, S. (2009). A Monte Carlo Comparison of the Power of the Test for Non-Linearity in Asymmetric Price Transmission Models. International Journal of Computational Intelligence Research and Applications, Vol. 3, No. 2: 281-283

Cook, S., Holly, S. and Turner, P. (2000). The power of tests for non-linearity: The Escribano-Pfann Model. Computational Economics, vol. 15: 223-226

Cook, S., Holly, S and Turner, P. (1999). The power of tests for non-linearity: the case of Granger-Lee asymmetry. Economics Letters, vol. 62: 155-59

Cook, S. (2003). A sensitivity analysis of threshold determination for asymmetric error correction models. Applied Economic Letters, vol. 10: 611-616
Granger, C.W. J and Lee, T.H. (1989). Investigation of production, sales and inventory relationships using multicointegration and non-symmetric error correction models. Journal of Applied Econometrics, vol. 4: 135- 159

Holly, S., Turner, P and Weeks, M. (2003). Asymmetric adjustment and bias in estimation of an equilibrium relationship from a co-integrating regression. Computational Economics, vol. 21: 195-202

Von Cramon-Taubadel , S. (1998). Estimating asymmetric Price Transmission with the Error Correction Representation: An Application to the German Pork Market", European Review of Agricultural Economics, 25, pp. 1-18. 